\title{
Limitations on Thickness of Absorber Layer in CdS/CdTe Solar Cells
}

\author{
T.I. Mykytyuk, V.Ya. Roshko, L.A. Kosyachenko and E.V. Grushko \\ Chernivtsi National University, 58012 Chernivtsi, Ukraine
}

\begin{abstract}
Calculations of the integrated absorptive capacity of CdTe layer taking into account the spectrum of the AM1.5 solar radiation and the absorption coefficient of CdTe are carried out. The recombination losses at the front and rear surfaces of the CdTe layer and in the space-charge region are also calculated based on the continuity equation. The restrictions on the thickness of CdTe in CdS/CdTe heterojunction have been ascertained taking into account all types of losses. It is shown that in CdTe, the almost complete absorption of photons (99.9\%) in the $h \nu>E_{\mathrm{g}}$ range is observed at a layer thickness of more than 20-30 $\mu \mathrm{m}$, and the absorptive capacity of photons in a CdTe layer of thickness $1 \mu \mathrm{m}$ is about $93 \%$. The obtained results indicate that when the CdTe absorber layer is very thin, it is impossible to avoid a noticeable decrease of the short circuit current density $J_{\text {sc }}$ as compared with a typical thickness of the absorber layer. The loss in $J_{\mathrm{sc}}$ is $19-20 \%$ when the thickness is $0.5 \mu \mathrm{m}$ compared to $5 \%$ for a thickness of $2-3 \mu \mathrm{m}$.
\end{abstract}

PACS: 84.60.Jt, 85.60.Dw, 73.61.Ga

\section{Introduction}

During the last decade the growth rate of solar industry based on thin-film CdTe solar cells is higher than any other photovoltaic (PV) technologies. One limiting factor for the terawatt production of CdTe modules in the future may be the scarcity of tellurium, which is extracted as a by-product in the production of copper. Exploration of tellurium-rich ores was not conducted since it was not necessary, but it is likely that this problem will be overcome. Parallel ideas are put forward to use ultrathin CdTe films in solar modules. In papers devoted to the CdS/CdTe solar cells with thin CdTe layer, there are discussions on the limitations imposed on the device efficiency. However, the literature does not pay adequate attention to important factors such as decrease in absorptive capacity, recombination losses at the surfaces and in the space charge region (SCR) of $p-n$ heterojunction. The results of consideration of these issues are presented in this paper.

\section{Absorptive capacity of CdTe}

Before the radiation reaches the $\mathrm{CdTe}$ absorber layer certain part of the radiation is reflected from the front surface of solar cell, the interfaces between the glass substrate, the transparent conductive oxide (TCO) layer, the $\mathrm{CdS}$ film and the CdTe layer. In addition to reflections, there is absorption in all layers. All these losses can be taken into account by the introduction of the factor $T(\lambda)$ in the expression for the integrated absorptivity of solar radiation of a CdTe layer with a thickness $d$ :

$$
A_{\Phi}(d)=\frac{\sum_{i} T(\lambda) \Phi_{i}\left[1-\exp \left(-\alpha_{i} d\right)\right] \Delta \lambda_{i}}{\sum_{i} T(\lambda) \Phi_{i} \Delta \lambda_{i}},
$$

where $\alpha_{i}$ is the absorption coefficient of CdTe at wavelength $\lambda_{i}, \Phi_{i}$ is the spectral power density of AM1.5 radiation, $\Delta \lambda_{i}$ is the interval between adjacent wavelengths in the table of ISO 9845-1:1992 [1]. The summation in Eq. (1) should be taken from $\lambda=300 \mathrm{~nm}$ to $\lambda=\lambda_{\mathrm{g}}=h c / E_{\mathrm{g}}$, where $E_{\mathrm{g}}$ is the band gap of CdTe $(1.47-1.48 \mathrm{eV})[2]$.

For $\alpha$ we can use the data given in Ref. [3] and supplemented by the results of our measurements at the absorption edge [2]. The optical transmission $T(\lambda)$ of the glass/TCO/CdS structure was taken from Ref. [4]. Figure 1a shows the absorptivity of solar radiation $A_{\Phi}$ as a function of the thickness of CdTe calculated by Eq. (1).

The solid line in Fig. 1a shows how the absorptivity of solar radiation power increases with increasing the thickness of CdTe. Obviously, it is more correct to deal with the flow of photons rather than the power of solar radiation. The expression for the absorptivity of the photon flux $A_{h \nu}$ has the form

$$
A_{h \nu}(d)=\frac{\sum_{i} T(\lambda) \frac{\Phi_{i}}{h \nu_{i}}\left[1-\exp \left(-\alpha_{i} d\right)\right] \Delta \lambda_{i}}{\sum_{i} T(\lambda) \frac{\Phi_{i}}{h \nu_{i}} \Delta \lambda_{i}} .
$$

The dependence of $A_{h \nu}$ on the thickness of CdTe is shown in Fig. 1a by the dashed line. As one can see the total photon absorption in CdTe occurs at $d=60-70 \mu \mathrm{m}$, although in a number of papers it is assumed that this occurs at a thickness of several $\mu \mathrm{m}$.

It should be borne in mind that a metal electrode is applied to the rear surface of the CdTe layer so that radiation is reflected from such an interface. In the case of $100 \%$ reflectance from the rear surface the absorptivity 

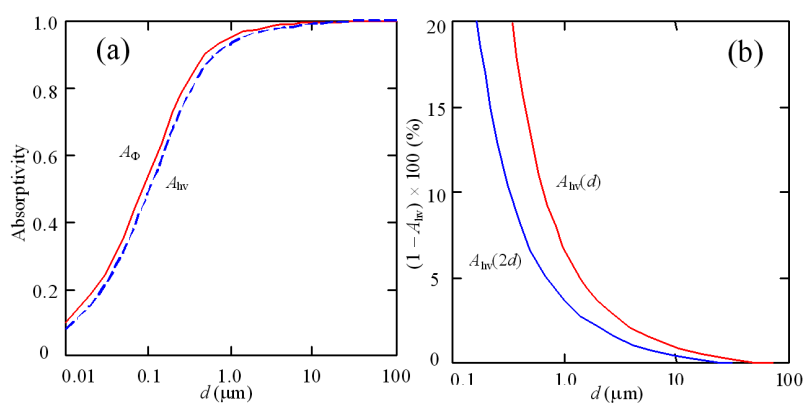

Fig. 1. (a) CdTe absorptivities of solar radiation power $A_{\Phi}$ and photon flux $A_{h \nu}$ in the solar radiation AM1.5 $\left(h \nu \geq E_{\mathrm{g}}\right)$. (b) Optical losses due to incomplete absorption of the solar radiation. Curves $A_{h \nu}(d)$ and $A_{h \nu}(2 d)$ are without and with taking into account reflections from the back surface, respectively.

is the same as for the double thickness of the absorber layer $A_{h \nu}(2 d)$. The calculation results in Fig. 1b show that in this case to ensure complete absorption of photons, the CdTe layer should be $40-50 \mu \mathrm{m}$. The absorptivity in CdTe with $d=1 \mu \mathrm{m}$ is about $97 \%$ and $\approx 93 \%$ at $d=0.5 \mu \mathrm{m}$.

\section{Recombination losses}

A quantitative description of losses caused by recombination can be made by calculating the short circuit current density $J_{\mathrm{sc}}$ :

$$
J_{\mathrm{sc}}=q \sum_{i} \eta\left(\lambda_{i}\right) \frac{T\left(\lambda_{i}\right) \Phi_{i}\left(\lambda_{i}\right)}{h \nu_{i}} \Delta \lambda_{i},
$$

where $q$ is the electron charge, $\eta\left(\lambda_{i}\right)$ is the photoelectric quantum efficiency at wavelength $\lambda_{i}$.

Quantum efficiency includes the drift and diffusion components, $\eta_{\text {drift }}$ and $\eta_{\text {dif }}$, respectively. The expressions for $\eta_{\text {drift }}$ and $\eta_{\text {dif }}$ can be used from Ref. [5]. These expressions contain the velocities of recombination at the front and rear surfaces of the CdTe layer $S_{\mathrm{f}}$ and $S_{\mathrm{b}}$, the height of the barrier at the contact with $\operatorname{CdS} \varphi_{0}$, and the SCR width

$$
W=\sqrt{\frac{2 \varepsilon \varepsilon_{0}\left(\varphi_{0}-q V\right)}{q^{2}\left(N_{\mathrm{a}}-N_{\mathrm{d}}\right)}},
$$

where $\varepsilon$ is the permittivity of the semiconductor, $\varepsilon_{0}$ is the electric constant, $V$ is the applied voltage, $N_{\mathrm{a}}-N_{\mathrm{d}}$ is the concentration of uncompensated acceptors in the CdTe layer.

\subsection{Recombination losses at front surface of CdTe layer}

Figure 2 shows the dependence of $J_{\mathrm{sc}}$ in $\mathrm{CdS} / \mathrm{CdTe}$ heterostructure on the width of the SCR $W$ calculated using Eq. (3) under the conditions of AM1.5. The carrier lifetimes are assumed to be $10^{-10}, 5 \times 10^{-10}$, and $2 \times 10^{-9} \mathrm{~s}$, based on the fact that these values in thin-film CdTe are usually in the range from $10^{-10}$ to $2 \times 10^{-9} \mathrm{~s}$ [6-8]. The mobilities of electrons and holes in thin-film
CdTe were taken to be 320 and $40 \mathrm{~cm}^{2} /(\mathrm{Vs})$, respectively $[9,10]$. The thickness of CdTe layer $d$ is accepted to be $10 \mu \mathrm{m}$, so that the thickness $d$ and the rear surface recombination $S_{\mathrm{b}}$ have practically no effect on these calculation results.
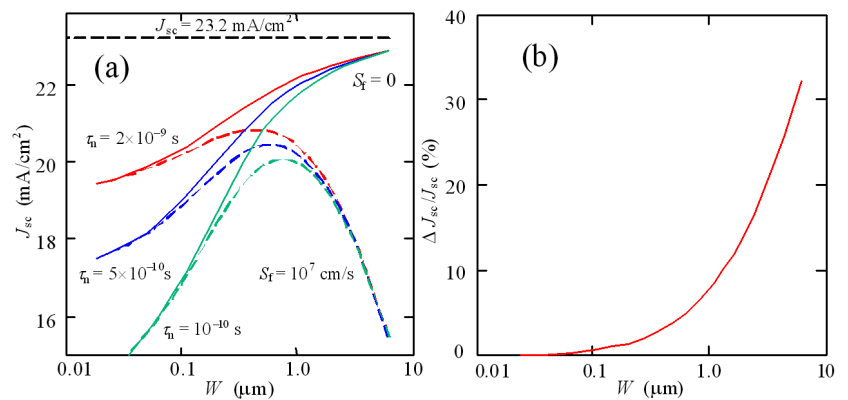

Fig. 2. (a) The dependence of short circuit current density $J_{\mathrm{sc}}$ on the width of the space-charge region $W$. (b) Recombination losses at the front surface in percentage at $S_{\mathrm{f}}=10^{7} \mathrm{~cm} / \mathrm{s}$.

Figure 2a shows that $J_{\mathrm{sc}}$ increases with $W$ in the range, where $W$ is well below $1 \mu \mathrm{m}$. This is because with an increase of $W$ more and more of the radiation is absorbed in the SCR. For $W>10 \mu \mathrm{m}$ and $S_{\mathrm{f}}=0, J_{\mathrm{sc}}$ continues to increase approaching the maximum possible value of $23.2 \mathrm{~mA} / \mathrm{cm}^{2}$, which corresponds to absorption in the SCR of almost all radiation passing through a glass substrate, $\mathrm{SnO}_{2}: \mathrm{F}$ and $\mathrm{CdS}$ layers. When the surface recombination is taken into account and $d>0.2-0.3 \mu \mathrm{m}$, the increase in $J_{\mathrm{sc}}$ due to the SCR expansion becomes slower and decreases with a further increase in $W$, since the electric field in the SCR is reduced and recombination at the front surface of CdTe is intensified. With the presence of surface recombination, maxima on the curves appear, when $W=0.4-0.8 \mu \mathrm{m}$.

To compare the effect of surface recombination with other kinds of losses, reduction of $J_{\mathrm{sc}}$ is convenient to be represented in percentages, which is shown in Fig. $2 \mathrm{~b}$ (the losses in percentage do not depend on the carrier lifetimes).

\subsection{Recombination losses in the $S C R$}

Recombination of photogenerated charge carriers in the SCR can be taken into account, using the Hecht formula [11]:

$$
\begin{array}{r}
\eta_{\mathrm{H}}(x)=\frac{\lambda_{n}}{W}\left[1-\exp \left(-\frac{x}{\lambda_{n}}\right)\right] \\
+\frac{\lambda_{p}}{W}\left[1-\exp \left(-\frac{W-x}{\lambda_{p}}\right)\right],
\end{array}
$$

where $x$ is the coordinate, where an electron-hole pair arises, $\lambda_{n}=\mu_{p} F \tau_{n}$ and $\lambda_{p}=\mu_{n} F \tau_{p}$ are the mean drift length of electrons and holes, respectively, $F$ is the electric field strength.

In the SCR, an electric field is not uniform, but consideration of the nonuniformity is simplified, since the field 
strength decreases linearly with $x$. The field strength $F$ in the expressions for $\lambda_{p}$ and $\lambda_{n}$ can be replaced by the mean values of $F$ in the sections $(0, x)$ and $(x, W)$ for electrons and holes, respectively [12].

Evidently, charge collection efficiency in the SCR is determined by

$$
\eta_{\text {coll }}=\int_{0}^{W} \eta_{\mathrm{H}}(x) \alpha_{\gamma} \exp \left(-\alpha_{\gamma} x\right) \mathrm{d} x,
$$

where $\alpha \exp (-\alpha x)$ is the generation rate of electron-hole pairs in terms of an incident photon.

Figure 3 shows the losses due to recombination in the SCR. As can be seen, the losses decrease rapidly with narrowing the SCR and increasing the carrier lifetimes. At the maximum of the dependence $J_{\mathrm{sc}}(W)$ in Fig. 2a $(W=0.4-0.5 \mu \mathrm{m})$, the recombination losses do not exceed $3-4 \%$ at $\tau=2 \times 10^{-9}$ s. If the SCR is not narrow enough and the carrier lifetimes is small (e.g., $10^{-10} \mathrm{~s}$ ), unacceptably high losses take place due to recombination in the SCR.

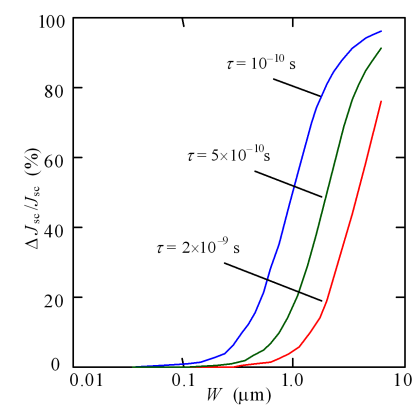

Fig. 3. Reduction of short-circuit current density caused by recombination in the SCR as a function of its width.

\subsection{Losses due to decrease in the thickness of CdTe layer}

As can be seen from Fig. 2a, taking into account the recombination at the front surface of the CdTe layer at the carrier lifetime $2 \times 10^{-9} \mathrm{~s}$, a maximum in the dependence of $J_{\mathrm{sc}}$ on $W$ is observed at $W=0.4-0.5 \mu \mathrm{m}$. These results were obtained with CdTe layer thickness $d=10 \mu \mathrm{m}$. Having fixed the $W$ value and the recombination velocity at the rear surface of the CdTe layer $S_{\mathrm{b}}=10^{7} \mathrm{~cm} / \mathrm{s}$, one can consider how $J_{\mathrm{sc}}$ will change with decreasing the film thickness $d$.

Figure 4 shows the results of calculations of the $J_{\mathrm{sc}}(d)$ dependences at $W=0.4,0.6,0.8$, and $1 \mu \mathrm{m}$ expressed in percents.

As can be seen, reduction of $J_{\mathrm{sc}}$ with decreasing $d$ begins to appear when $d$ is less than $\approx 5 \mu \mathrm{m}$, and the effect is enhanced, when the value of the thickness $d$ approaches to $W$. One can state that thinning the CdTe layer to $1 \mu \mathrm{m}$ reduces $J_{\mathrm{sc}}$ by about $5 \%$, and when $d$ approaches to $W=0.4 \mu \mathrm{m}, J_{\text {sc }}$ decreases by $\approx 12 \%$.

It also follows from Sect. 2 that at $d$ less than a few $\mu \mathrm{m}$ the losses associated with the lack of absorptive capacity

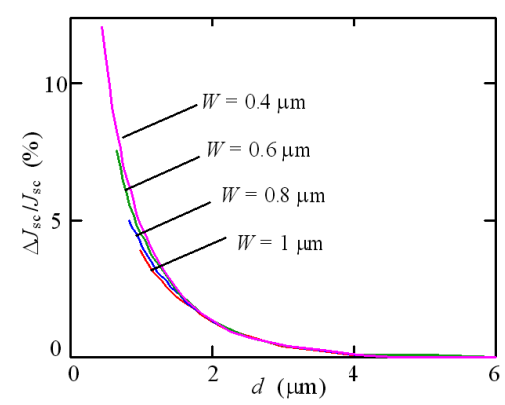

Fig. 4. Reduction of $J_{\mathrm{sc}}$ with $d$ of the CdTe film calculated for different widths of the SCR.

reveal themselves perceptibly. Figure 5 shows the dependence of $J_{\mathrm{sc}}$ on $d$ due to both the diminishing of a photoelectrically active layer and a decrease in absorptivity of the layer. As it turns out, reducing $J_{\mathrm{sc}}$ due to both kinds of losses in the CdTe layer is comparable: $1-2 \%$ for $d=2 \mu \mathrm{m}$ and $7-10 \%$ for $d=0.5 \mu \mathrm{m}$.

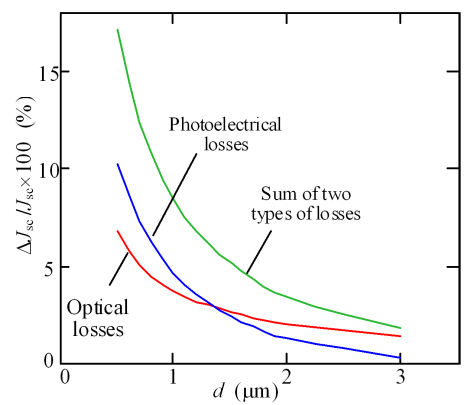

Fig. 5. Effect of both the diminishing of photoelectrically active part of the CdTe layer and the optical losses on the short circuit current $J_{\mathrm{sc}}$ as a result of reducing the thickness $d$ of the CdTe layer.

\section{Generalization of the calculation results}

First of all, it should be preferred to have a material with high carrier lifetime, i.e. $2 \times 10^{-9}$ s. Further, in trying to create the conditions so that major part of the radiation is absorbed in the SCR, one cannot extend the SCR more than $0.5-0.8 \mu \mathrm{m}$. As can be seen from Fig. 2a, the short-circuit current density $J_{\mathrm{sc}}$ decreases very slightly, when SCR width is reduced to $0.3 \mu \mathrm{m}$. Thus, the width of the SCR $W=0.3 \mu \mathrm{m}$ can be considered as nearly optimal. Such a choice of the $W$ value is not contrary to the requirements relating to the recombination losses in the SCR. So, having chosen the values of $\tau$ and $W$, one can consider the dependence of $J_{\mathrm{sc}}$ on the CdTe layer thickness $d$. Losses due to recombination at the front surface of the CdTe layer and in the SCR can be assumed to be 2.4 and $0.4 \%$, respectively.

Figure 6 shows the relative change of $J_{\mathrm{sc}}$ as a function of $d$ taking into account all types of losses. It is shown that when $d=2-3 \mu \mathrm{m}$ the total losses are about $5 \%$ 
larger than those for a thick layer but when thinning the CdTe layer up to $0.5 \mu \mathrm{m}$ the losses increase to $\approx 20 \%$.

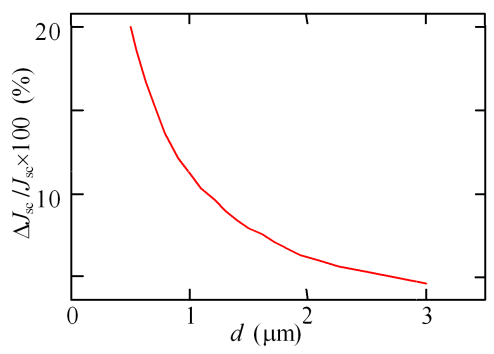

Fig. 6. Reduction of short circuit current with decreasing the thickness of the CdTe layer $d$ taking into account all types of losses.

\section{Conclusions}

The analysis on the thinning of CdTe layer and the consequent reduction in $J_{\mathrm{sc}}$ allow to formulate some important practical conclusions. (i) For the CdTe layer thickness of 2-3 $\mu \mathrm{m}$, the absorptivity of photons is about $97 \%$, and reduces to $\approx 93 \%$ at a layer thickness of $0.5 \mu \mathrm{m}$. (ii) In thin-film CdTe with the lifetimes of the charge carriers of $(0.1-2) \times 10^{-9} \mathrm{~s}$, the dependence of short circuit current density $J_{\mathrm{sc}}$ on $W$ follows a curve with a maximum at $W=0.4-0.8 \mu \mathrm{m}$. (iii) If the SCR is sufficiently narrow ( $W<0.3-0.4 \mu \mathrm{m})$, the lowering of short circuit current density $J_{\mathrm{sc}}$ due to the recombination of photogenerated carriers in the SCR does not exceed $0.4 \%$. (iv) Thinning of the CdTe layer to $1 \mu \mathrm{m}$ reduces $J_{\mathrm{sc}}$ by $\approx 5 \%$, and when $d$ approaches to $0.5 \mu \mathrm{m}, J_{\mathrm{sc}}$ decreases up to $\approx 20 \%$.

\section{Acknowledgments}

The study was supported by the State Foundation for Fundamental Investigations of Ukraine within the project $\Phi 40.7 / 014$.

\section{References}

[1] Reference Solar Spectral Irradiance at the Ground at Different Receiving Conditions, Standard of International Organization for Standardization ISO 9845-1:1992.

[2] L.A. Kosyachenko, V.M. Sklyarchuk, O.V. Sklyarchuk, O.L. Maslyanchuk, Semiconductors 45, 1247 (2011).

[3] T. Toshifumi, S. Adachi, H. Nakanishi, K. Ohtsuka, J. Appl. Phys. 32, 3496 (1993).

[4] L.A. Kosyachenko, E.V. Grushko, X. Mathew, Solar Energy Mater. Solar Cells 96, 231 (2012).

[5] L. Kosyachenko, G. Lashkarev, E. Grushko, A. Ievtushenko, V. Sklyarchuk, X. Mathew, P.D. Paulson, Acta Phys. Pol. A 116, 862 (2009).

[6] A.L. Fahrenbruch, in: Conf. Record of the IEEE 4 th World Conf. on Photovoltaic Energy Conversion, Waikoloa (USA), 7-12 May 2006, Ed. S.G. Bailey, Waikoloa 2006, p. 376.

[7] J. Sites, J. Pan, Thin Solid Films 515, 6099 (2007).

[8] T.A. Gessert, W.K. Metzger, P. Dippo, S.E. Asher, R.G. Dhere, M.R. Young, Thin Solid Films 517, 2370 (2009).

[9] M. Gloeckler, A.L. Fahrenbruch, J.R. Sites, in: Proc. 3rd World Conf. on Photovoltaic Energy Conversion, Osaka (Japan), Vol. 1, Ed. K. Kurokawa, Arisumi, Osaka 2003, p. 491.

[10] M.S. Hossain, N. Amin, M.A. Matin, M.M. Aliyu, T. Razykov, K. Sopian, Chalcogen. Lett. 8, 263 (2011).

[11] K. Hecht, Zeits. Phys. 77, 235 (1932).

[12] L.A. Kosyachenko, E.V. Grushko, V.V. Motushchuk, Solar Energy Mater. Solar Cells 90, 2201 (2006). 